\title{
XXXIX. The effect of interionic force in electrolytes
}

\section{S.R. Milner D.Sc.}

To cite this article: S.R. Milner D.Sc. (1918) XXXIX. The effect of interionic force in electrolytes , Philosophical Magazine Series 6, 35:208, 352-364, DOI: 10.1080/14786440408635771

To link to this article: http://dx.doi.org/10.1080/14786440408635771

册 Published online: 08 Apr 2009.

Submit your article to this journal 중

Џll Article views: 3

Q View related articles $\square$

Citing articles: 1 View citing articles $\square$ 
reduced to a very small amount. Each stoma is fully efficient in proportion to its radius; but the output will be diminished for all sizes of stoma because it has to transpire into a nearly saturated space. When the stoma becomes constricted it operates more feebly, but under better conditions; if a circular stomatal aperture of dimensions as above is constricted to $\frac{1}{7}$ of its radius, its own resistance will be half the total resistance to transpiration instead of only $\frac{1}{8}$; if it is constricted to $\frac{1}{5}$, the resistance of the stoma itself will be $\frac{7}{8}$ of the whole, and the layer of moist air ontside will hardly count. The question is whether we can assume a still layer of air anything like $1 \mathrm{~mm}$. thick. As moist air is sensibly lighter than dry, such a layer could hardly be establishod if the leaf itself is not quite still, unless possibly to some degree on the lower surface of a horizontal leaf.

The problem whether a cause like this, which I take to be the essence of Dr. Jeffroys's important suggestion, has really intervened in observations such as those quoted by Darwin from F. E. Lloyd (p. 277) could only be probed by further experiment under suitable precautions.

The question whether the sap-current in trees is reduced on still days is much simpler, for the whole region of the tree-top may become nearly saturated.

These considerations, which apply to the transpiration of vapour from the stomata, are pertinent, of course, equally to the diffusion of carbon dioxide into them. They seem directly to confirm, from a different aspect, the conclusions of Brown and Escombe that the stomatal cavities are capable of much more absorption than they are called upon to perform.

Cambridge, March 9.

XXXIX. The Effect of Interionic Force in Electrolytes.

$B y$ S. R. Milner, D.Sc.*

PART II.

Ionic Mobility and Osmotic Pressura.

TTHE first attempt to determine the effect of interionic 1 force on the ionic mobility is due to Sutherland $t$, whose method is based on the following idea :-The effect of the forces will approximate to what would be obtained if the ions were regularly distributed throughout the liquid, say at the centres of equal cubes. When they are displaced

* Communicated by the Author.

$\dagger$ Phil. Mag. xiv. j. 1 (1907). 
from these positions by an applied electric field, the interionic forces act as restoring forces in a way which would give rise to a sort of rigidity of the ionic configuration were it not for the fact that the "actions which produced the original uniformity" (thermal motions?) will cause the rigidity to be continually breaking down. The process of breaking down originates a special type of viscosity, which acts in addition to ordinary viscosity when conduction is taking place. A second type of viscosity due to the polarization of the medium is also discussed, and the conclusion is reached that when these viscosities are taken into consideration the diminution of $\lambda$, with increase in the concentration, can be accounted for without any association of the ions into molecules taking place.

Sutherland's calculation does not bring the conductivity variation into any relation with that of the freezing-point; and it is based on several speculative hypotheses which are not always convincing. This is particularly the ease in regard to the assumed configuration of the ions; the special type of regularity of this is a feature which is inconsistent with the general theory of the distribution of ions to which the kinetic theory leads.

Effect of permanence of the distribution on the mobility. -The method of calculation adopted here is based on the assumption that the distribution of the ions remains undisturbed in the interior of the electrolyte when a current is being carried. Suppose we have a mixture of positive and negative ions contained in a volume, and in the first place suppose that they are subject to no interionic forces. We must assume that they are distributed at random throughout the volume, for there are no data for assuming anything else. Now suppose that an external electric field is applied which gives each positive ion a velocity to the right, and each negative jon one to the left. In the interior of the voiume the random distribution will not be disturbed, as is easily seen whether the velocities are all equal or whether they vary arbitrarily from ion to ion.

When interionic forces are present the distribution is no longer random. It becomes modified in such a way that the chance of a positive ion being found in a given position will depend on the mutual potential energy which it possesses in that position with the other ions. If we now imagine all the positive ions displaced to the right and the negative ones to the left--with the same or with arbitrary velocities-the distribution will be disturbed. It will, in fact, tend to be converted into a random distribution. Consequently we see 
that, if the distribution is to remain permanent when the electrolyte is carrying a current, the velocity with which each ion must be supposed to move under the influence of the applied electric field must be a function at each instant of the mutual potential energy which the ion possesses with the others.

The way in which the random distribution will be modified when the ions are subject to interionic force is given by a theorem due to Boltzmann. Let us suppose that we take a large number of instantaneous views of a certain region of the liquid, and that in each view we observe the positions and signs of all the ions which are present in it. We will confine our attention in the first place to those views alone, $n$ in number, in which the region contains $m$ ions, $A_{1} \ldots A_{m}$, and no more, and we will suppose that these are all so far away from the ions outside the region that the forces between the ions inside and outside are negligible. This will simplify the statement of the argument without affecting the generality of it in any way, since the region may, if necessary, comprise the whole of the liquid. In a certain number, say $\nu$, of the $n$ views, the $m$ ions will be found in small equal volumes $d v_{1} \ldots d v_{m}$, situated at the points $\mathrm{P}_{1} \ldots \mathrm{P}_{m}$. For shortness we will call this the $\mathrm{P}$ configuration, and speak of the ion $A_{1}$ as "occupying the position" $\mathrm{P}_{1}$, the uniform size of the elementary volumes being understood. In another number $\nu^{\prime}$ of views the ions will be found in positions $\mathrm{P}_{1}^{\prime} \ldots \mathrm{P}_{m}^{\prime}$ ( $\mathrm{P}^{\prime}$ configuration $)$. On a purely random distribution we should have

$$
\nu=\nu^{\prime},
$$

but in the modification caused by the presence of interionic force,

$$
\nu=\nu^{\prime} e^{-\left(\phi-\phi^{\prime}\right) / k \mathrm{~T}}, \quad . \quad \text {. }
$$

$\nu / \nu^{\prime}$ here stands for the probability of the $\mathrm{P}$ configuration relative to that of the $\mathrm{P}^{\prime} . \phi$ and $\phi^{\prime}$ are the respective mutual potential energies of the ions in each configuration, $i$. e. the work done by the system when the ions are moved to infinite distances apart. When the forces are attractive $\phi$ and $\phi^{\prime}$ are negative quantities. $k \mathrm{~T}=\frac{2}{3} \times$ average translatory kinetic energy of an ion.

Equation (5) can easily be transformed so as to represent the absolute probability of a given configuration (estimated under the special conditions attached to the total number of views $n$ ) by writing it in the form

$$
\nu=\mathrm{K} n e^{-\phi / k \mathrm{~T}} d v_{1} \ldots d v_{m} . \quad . \quad . \quad . .
$$


The essential feature of the ionic distribution represented by (6) is of course its permanence, that is, that it is undisturbed by the thermal motions of the ions. Let us suppose that the $n$ views were taken at certain times $t_{1} \ldots t_{n}$. If we were to take another set of views at times $t_{1}+\tau, \ldots t_{n} \vdash \tau$, where $\tau$ is a very small time, we should find the same number of views in which the ions have the configuration $\mathrm{P}$; the actual views will not be identical in the two cases, in some of the $n$ views at $t$ the ions will have left the volumes $d v$, but in an equal number of other cases ions will have come into them.

Now suppose that the electrolyte is under the influence of an external electric field when these two sets of views are being taken. In addition to the thermal displicement which each ion undergoes in each of the times $\tau$, it will be dragged by the electric field a certain distance to the right or left. We can effect a considerable simplification by observing that, since the thermal displacements do not affect the distribution, we can imagine them to be non-existent without affecting in any way the result of reasoning concerning the effect on the distribution of the displacements due to the electric field. With this simplification the problem to be solved becomes this :-A set of views at times $t_{1}, \ldots t_{n}$, and another set at $t_{1}+\tau, \ldots t_{n}+\tau$, being taken of a system of ions existing in configurations the probability of which is given by $(6)$ and at rest during the intervals $\tau$ so far as thermal motions are coneerned, but in which each ion is dragged during the intervals by an external force to right or left according as it is positive or negative, what is the average velocity with which an ion such as $A_{1}$ in a given position must move in order that the distribution may not be disturbed? By the average velocity is meant the average for all the views which show the ion in the given posilion, but as it makes no difference whether it varies from view to view or is uniform, we may in the calculation treat it as uniform.

Let $u_{1}, \ldots u_{m}$ be the velocities of each ion $\mathrm{A}_{1}, \ldots \mathrm{A}_{m}$. Describe small cylinders of area $a$ and of arbitrary lengths $d x_{1}, \ldots d x_{m}$ near each of the points $\mathrm{P}_{1}, \ldots \mathrm{P}_{m}$ to the right or left of the points according to the sign of the ion. The number of cases in which an ion is to be found in each of these cylinders will be the same in the views at $t+\tau$ as it is in the views at $t$. Consequently the number $\nu_{1}$ of views in which an ion will enter each cylinder during the intervals $\tau$ is the same as the number $\boldsymbol{y}_{2}$ in which an ion will leave each cylinder. But $\nu_{1}$ comprises all those views in which the $m$ ions are 
situated in the infinitesimal volumes $a u_{1} \tau, \ldots a u_{m} \tau$ at $\mathrm{P}_{1}, \ldots \mathrm{P}_{m}$, or

$$
\nu_{1}=\mathrm{K} n e^{-\Phi / k \mathrm{~T}} \alpha u_{1} \tau \ldots \alpha u_{m} \tau .
$$

A similar expression holds for $v_{2}$ except that both $\phi$ and the $u$ 's are infinitesimally different:

$$
\begin{aligned}
\nu_{2} & =\mathrm{K} n\left\{e^{-\Phi / k \mathrm{~T}}+\frac{d}{d x_{1}} e^{-\Phi / k \mathrm{~T}} d x_{1} \ldots+\frac{d}{d x_{m}} e^{-\phi i k \mathrm{~T}} d x_{m}\right\} \\
& \times a\left(u_{1}+\frac{d u_{1}}{d x_{1}} d x_{1}\right) \tau \ldots a\left(u_{m}+\frac{d u_{m}}{d x_{m}} d x_{m}\right) \tau .
\end{aligned}
$$

Equating $\nu_{1}$ and $\nu_{2}$ we get

$$
\frac{d}{d x_{1}}\left\{e^{-\Phi / k \mathbf{T}} u_{1}\right\}=\ldots=\frac{d}{d x_{m}}\left\{e^{-\phi_{i} k \mathbf{T}} u_{m}\right\}=0 .
$$

Now, if we write $\phi$ in the form

$$
\phi=\phi_{1}+\phi_{1}{ }^{\prime}=\phi_{2}+\phi_{2}{ }^{\prime} \text {, }
$$

where $\phi_{1}$ is the mutual energy of $A_{1}$ with all the rest, $\phi_{1}^{\prime}$ the mutual energy of all the $m-1$ ions other than $A_{1}, \phi_{2}$ that of $A_{2}$ with all the rest, \&c., we see that the only part of $\phi$ which is affected by $d / d v_{1}$ is $\phi_{1}$, and similarly for each of the other ions. Consequently, integrating (7), we see that the conditions which must be satisfied in order that the distribution may not be disturbed are

$$
\begin{aligned}
& u_{1} e^{-\Phi_{1} / k \mathbf{T}}=\text { const., } \\
& u_{2} e^{-\Phi_{2} / k \mathbf{T}}=\text { const., \&c. }
\end{aligned}
$$

The constant is independent of $x$, that is, of $\phi_{1}, \phi_{2}$, \&c., and is equal to the velocity with which an ion will move when it is so far away from the others that its mutual energy with them is zero. In these circumstances the velocity will be conditioned simply by the friction of the water, and it is clearly the same for all ions of the same sign. Calling it $u_{0}$ in unit field, we shall then have for the mobility $u_{1}$, which an ion must be reckoned to possess when it exists in a place where its mutual energy with other ions is $\phi_{1}$,

$$
u_{1}=u_{0} e^{\phi_{1} / k \mathbf{T}} \text {. }
$$

Effect on Osmotic Pressure.-In the method of the kinetic theory of considering the pressure of a gas as the rate at which momentum is transferred through a unit plane within it, a careful distinction must be made between "internal" and "external" pressure. Consider a gas in which-say by impressed mochanical forces-the potential energy of a 
molecule when in a certain region $\mathrm{R}$ is $\phi$ (a negative quantity) and zero elsewhere. The distribution will be such that the chance of a molecule occupying a position inside $R$ is to that of its occupying one outside as $e^{-\Phi / k T}: 1$, and, in fact, the densities in the two parts will adjust themselves in this proportion. If we imagine a unit plane situated inside $R$, the momentum transferred through it per second will be the total pressure inside $R$, but it is only a certain fraction$e^{+\phi i k \mathbf{T}}$-of the molecules passing through the plane which are capable of transferring their momentum outside the region. We can thus divide the pressure in $R$ into two parts-the external pressure, which is due to momentum capable of being transferred outside it, and which is, in fact, in equilibrium with the pressure outside, and the internal pressure, which in this case will be exerted on the mechanical constraints which cause the increased density in $R$.

A similar state of things occurs when we deal with a group of ions existing momentarily in a liquid. The whole momentum passed per second by the ions of the group through a unit area drawn in the interior of the group will not be delivered to places of zero potential energy, and the fraction of it that is so delivered will be in statistical equilibrium with the pressure exerted by those ions which are in positions of zero energy. We may call this fraction the external pressure $p$ of the ions in the group or the pressure of the "free" ions-understanding by free ions those which momentarily have no mutual energy with any others. Let us inquire how much of the momentum of an ion existing in a group such as that considered above would, on the average, be capable of being transferred to a place of zero potential.

Consider a single ion of mass $m$ moving in a random direction with velocity $v$. The average rate at which it transfers, parallel to a given direction, the component in that direction of its momentum is $\frac{1}{3} m v^{2}$, or, if we take into the average all the possible velocities which it may have, $\frac{1}{3} m \bar{v}^{2}$ or $k \mathrm{~T}$. The sum of this quantity for every ion in the mixture gives the total (i. e. internal +external) pressure $x$ volume, $P V$, of the electrolyte. The contribution of each ion to the total $\mathrm{PV}$ is thus a scalar quantity $\frac{1}{3} m \overline{\eta^{2}}$ associated with the ion-these contributions will therefore obey the same law of distribution as do the ions themselves.

Consider now two configurations $\mathrm{P}$ and $\mathrm{P}^{\prime}$ of the group of $m$ ions dealt with above. In $\mathrm{P}$ the ions occupy the

Phil. Mag. S. 6. Vol. 35. No. 208. April 1918. 2 C 
positions $P_{1} \ldots P_{m}$, the mutual energy is $\phi=\phi_{1}+\phi_{1}{ }^{\prime}$, and the number of views in which it is found is

$$
\nu=\mathbf{K} n e^{-\Phi_{1}^{\prime} / k \mathrm{~T}} e^{-\Phi_{1} / k \mathrm{~T}} d v_{1} \ldots d v_{m} . . . .
$$

In $\mathrm{P}^{\prime}$ let $\mathrm{A}_{2}, \ldots \mathrm{A}_{n}$ be in the same positions as before, but let $A_{1}$ be in a place of zero mutual energy. The mutual energy of the group is now $\phi_{1}{ }^{\prime}$ and the number of views in which this configuration is found is

$$
\nu^{\prime}=\mathrm{K} n e^{-\Phi_{1}^{\prime} / 7 \mathrm{~T}} d v_{1} \ldots d v_{m} .
$$

In the series of $n$ views taken one after the other at arbitrary times the configuration of the system is changed between one view and the next by complex thermal motions. Omitting all the other views, let us confine our attention to thel $\nu$ views of the configuration $\mathrm{P}$ and the $v^{\prime}$ views of $\mathrm{P}^{\prime}$. These are observed at certain successive times and are all the views of these configurations which are observed in the series. In the intervals between them the configurations change over one into the other as the result of thermal motions, but not indiscriminately. It is only in a fraction $\nu^{\prime} / \nu$ of the $\nu$ views of the $\mathrm{P}$ configuration that a change by thermal motions into the $\mathrm{P}^{\prime}$ configuration will occur. On a random distribution the fraction would be unity. The change in the configuration considered consists simply in the transference by thermal motion of the ion $A_{1}$ from a position of mutual energy $\phi_{\mathrm{I}}$ to one of zero mutual energy. We see that, given the ion in this position, the probability that such a transference will take place is not the same as it would be on a random distribution (i.e. in the absence of interionic forces), but $\nu^{\prime} / \nu$ or $e^{\Phi_{1} / k \mathbf{T}}$ times as great ${ }^{*}$.

On a random distribution the whole of the scalar property ${ }_{3}^{1} m \overline{v^{2}}$ associated with each ion is capable of being transferred from one position to another. This gives a random, or on the large scale a uniform, distribution of the pressure throughout the volume. It follows from the preceding proposition that,

* The proposition is so far only proved for the case in which the $m-1$ ions other than $A_{1}$ remain fixed during the transference of $\mathbf{A}_{1}$. If these also undergo displacements we shall have a simultaneous alteration of $\phi_{1}$ and $\phi_{1}^{\prime}$. In so far as these displacements affect $\phi_{1}$ only, it is immaterial to the argument whether $A_{1}$ reaches a state of zero mutual energy by its own displacement or by suitable ones of the other ions, the expression for $\nu^{\prime} / \nu$ being the same in either case. If, on the other hand, they alter $\phi_{1}{ }^{\prime}$, the expression (9) shows that this is an event independent of the change in $A_{1}$ 's mutual energy, the probability of the simultaneous occurrence of the two events being the product of the two probabilities. The truth of the proposition is thus unaffected by all other ionic changes which may proceed simultaneously with the transference of the ion $A_{1}$ to a position of zero energy. 
when interionic force is present, the average amount of the property which is capable of being transferred from a position in which the mutual energy of its ion is $\phi_{1}$ to a position of zero mutual energy, is

$$
{ }_{3}^{1} m \overline{v^{2}} \cdot e^{\Phi_{1} / k \mathbf{T}} \text {. . . . . . . }
$$

This quantity represents the contribution to the external $p V$ made by the ion when it forms part of a group. The summation of it for each ion in the electrolyte would give the external $p \mathrm{~V}$ or, strictly speaking, its instantaneous value in the view observed. The external pressure of a system of ions subject to interionic force determined in this way is a perfectly definite thing everywhere in equilibrium throughout the volume. It is identical with the pressure of the free ions as defined above. Superposed on it in the interior of groups is the internal pressure (got by summing $\frac{1}{3} m v^{2}\left(1-e^{\Phi_{1} / R \mathrm{~T}}\right)$ for each ion), which is exerted against the mutual forces and is not effective on the walls.

A comparison of the result (10) for the contribution of an ion to the external $p \mathrm{~V}$ when it is in a position of mutual energy with others with that (8) for the mobility of the ion in the same circumstances, shows that both are affected by the mutual energy in exactly the same way. Suppose now we follow in imagination the history of an individual ion in an electrolyte for a long time. We shall observe that its state as regards the mutual energy $\phi_{1}$ which it possesses with other ions is continually varying. If we take the average value over a sufficiently long time of the quantities

$$
u_{0} e^{\Phi_{1} / k \mathrm{~T}} \text { and } k \mathrm{~T} \cdot \theta^{\Phi_{1} / k \mathrm{~T}} \text {, }
$$

we shall get in the first instance the average value of the mobility $u_{1}$ of the ion (which is of course the same as that $u$ of any other ion of the same kind, but $u_{0}$ differs if the kind differs), and in the second instance a quantity which, when multiplied by the total number $\mathrm{N}$ of ions in the electrolyte (combined or not) gives the product $p V$ of the pressure of the free ions into the volume. It is clear that, whatever the effect of $\phi_{1}$ may be on the actual values, we shall always have

$$
\frac{u}{p \mathrm{~V}}=\frac{u_{0}}{\mathrm{~N} k \mathrm{~T}}=\text { const. . . . . . }
$$

Hence in an electrolyte, while alterations with the concentration of $u$ and $p \mathrm{~V}$ may, and indeed will, be produced as the result of the presence of interionic forces, the alterations will always be such that the ratio of $u$ to $p V$ remains unaffected. 
PART III.

The theorem thus proved can be applied to throw some light on the nature of the interionic forces in strong electrolytes, but before applying it it is necessary to identify $u_{0}$ and $p$ clearly with measured physical quantities. As regards $u_{0}$ there is no difficulty : it represents the mobility of an ion in a region where it is free from interionic torce. $u_{0}$ is there conditioned only by the friction of the water, and can thus be identified with the exporimentally determined mobility at zero concentration to which the same condition applies. The case is different for $p$ the free ionic pressure. In what relation does this stand to the measured osmotic pressure? It will be useful to consider this point in connexion with three possible theories of the constitution of electrolytes.

(1) In the original theory of Arrhenius electric interionic forces are neglected, and an ion is assumed to be definitely either associated (when it contributes nothing to the free ionic pressure $p$ ) or free. Although $p$ is not susceptible to direct measurement, a clear conception of it can be got.

The general theorem is of course independent of the law of force between the ions and applies to this theory equally with others. Interionic forces (of the kind referred to below as "chemical" forces) produce an increase in the frequency of occurrence of ions in an associated state with a consequent reduction in the pressure of the free ions in the ratio of $1-\beta: 1$. The average mobility of an ion, taken over a period long enough to include its being combined as well as free, is reduced in the same ratio. This agrees with the experimental requirement of an equality in the freezingpoint and conductivity variations, for in calculating the complete osmotic pressure $P$, allowance must be made for the molecular pressure of the fraction $\beta / 2$ of associated ions. The reduction in $\mathrm{P}$ is thus in the ratio $\left(1-\beta+\frac{\beta}{2}\right): 1$, and consequently

$$
\frac{\tau}{\tau_{0}}=2-\beta, \quad \frac{\lambda}{\lambda_{0}}=1-\beta,
$$

in agreement with the experimental results ( $v$. Part I.).

The difficulty here, as already mentioned, is the failure of the mass action law (4) to represent correctly the variation of $\beta$. When we consider the dynamical assumptions on which (4) is based, we find it essential that the forces which tend to produce association of the ions must fall off very 
rapidly with the distance*. The law indeed forms a limiting case for which the field of force surrounding each ion is infinitely strong but confined to an infinitely thin shell. This means that the mass law (4) applies solely to association and dissociation which is the result of what we may call "chemical" forces, using this name to distinguish forces of this type from the electrical forces of the free ionic charges which fall off very slowly with the distance. If association is produced by these (4) will not apply to it.

(2) The preceding conclusion suggests that the failure of the mass law may be due to a mistaken view of the nature of the interionic forces which cause association. The apparent association may be partly-or wholly - due to the electric forces. In attempting to investigate this view the first difficulty is to know exactly what is the law of force between the ions. Are we to assume that every + ion attracts every - ion and repels every + ion in the liquid according to the inverse square law? If the mixture of ions wore a gaseous one this assumption would presumably be a sound one, but its validity is more doubtful in the case of an electrolyte, where the forces between the ions are affected by the intervening water molecules. As the first step, however, it seems the most straightforward assumption to make, and in previous papers $\dagger$ I have worked out by what is, I think, a strict method, the approximate effect on the osmotic pressure of a mixture of ions in which interionic force of this character is assumed to exist. The calculation, as might be expected from the complexity of the forces, is lengthy and need not be further referred to. The net effect of the interionic forces was found to give a reduction of the osmotic pressure which appears to be in accurate agreement with the experimentally found results for dilute aqueous solutions of strong binary electrolytes. In these cases therefore there is ground for believing that "chemical association," if existent, is extremely small, and that the effects observed are due entirely to the electrical interionie forces. Before we can apply to this view the proposition of the present paper, it is necessary to settle the relation in which the free ionic pressure $p$ stands to the measured osmotic pressure $P$. Unless they are different from each other, an inequality

* This is the kinetic aspect of the thermodynamical stipulation that the osmotic pressures of the ions must obey the perfect gas law. The assumption is formally made in Boltzmann's original deduction, and it can easily be shown that any other will result in a law different from (4).

† Phil. Mag. xxiii. p. 551 (1912); xxv. p. 742 (1913). 
between the freezing-point and conductivity variations will result from the proposition. For if $\beta$ is the fractional alteration of $p \mathrm{~V}$, and therefore of $u$, and if further $p=\mathrm{P}$, we shall get

$$
\frac{\tau}{\tau_{0}}=\frac{\mathrm{PV}}{\mathrm{RT}}=\frac{p \mathrm{~V}}{\overline{\mathrm{RT}}}=2(1-\beta) ; \frac{\lambda}{\lambda_{0}}=1-\beta,
$$

which is in conflict with the experimental result (3) (Part I.).

$p$ and $\mathrm{P}$, however, cannot be the same, as is evident from the following consideration :-Consider a pair of ions which happen to be fairly close together and under the influence of each other's attraction, and let a number of representative views be taken. In a certain fraction- $e^{\Phi_{1} / k T}-$ of the cases the ions contribute to the free ionic $p \mathrm{~V}$, in the remainder of the cases the ions act as though bound together and contribute nothing to $p \mathrm{~V}$, but in these cases the pair will make to the measured PV exactly the same contribution as if the ions formed an actual molecule. The effect of electrical forces is in this respect exactly similar to that produced by chemical association. The electrical bond it is true persists when the ions are well separated from each other, while the chemical bond acts only at very small distances, but this difference is immaterial in considering the molecular pressure which a pair of bound ions will exert. For the type of force considered, however, the bonds are not confined to single pairs of ions, but each pair must be considered as forming part of a large group, with the rest of the ions in which it possesses a certain mutual energy, and so is not free to exercise its full molecular pressure. While a difference between $p$ and $P$ may on these lines be inferred to oxist, it is difficult to settle exactly what it is *. It seems doubtful that it would be such as to give the exact equality between $\beta_{1}$ and $\beta_{2}$ which experiment suggests unless the mutual energy between each pair and the rest of the group is negligible.

(3) A theory which is to some extent intermediate between (1) and (2) has much to recommend it. We must infer from the preceding comparisons that the interionic forces must extend over considerable distances, as it is only

- The straightforward way to settle this point is to calculate $p$ by a method in accordance with its definition on $p .357$ and compare it with $P$. The calculation can be carried out strictly by exactly the same method as that by which $\mathrm{P}$ was originally determined (loc. cit.). It has been done, but unfortunately the numerical results in both cases can only be obtained in an approximate form which is not sufficiently accurate to determine definitely what is the difference between them. 
in this way that a satisfactory explanation of the failure of the mass action law can be got. On the other hand, the idea of the molecular pressure of a pair of associated ions seems also necessary to obtain an aceurate agreement with the experimental equality of the freezing-point and conductivity variations. Both conditions will be satisfied if the law of interionic force be such as practically to confine the electrical attraction to pairs of nearest ions. Now, the view that in an electrolyte each + ion attracts every - ion and repels every + ion is undoubtedly a highly artificial one. How artificial it is is made evident by observing that the mutual energy of an ion with others would have to be expressed as a sum of hundreds of terms before any close approximation to its value could bo obtained. These represent the mutual energies with the nearest ion, the next nearest, the third nearest, \&c., and form terms which partly cancel each other as the successive ions are + and It is unlikely that this state of things represents a physical reality. Indeed, the assumption on which it is based, that the action of the water molecules cin be simulated by that of a continuous medium of S.I.C. the same as that of water in mass, is hardly likely to be true. It is probably a good deal nearer to the truth to imagine surrounding each ion a number of polarized water molecules which tend to form chains linking together pairs of temporarily nearest oppositely charged ions. Such an action would not be the same as that of a uniform medium; it would be more analogous to the action of iron filings in forming chains between two magnetic poles. The general effect would be to increase the attraction between an ion and the nearest one to it of unlike sign at the expense of the attraction of more distant ones, which latter might well be negligible in consequence.

The view of the constitution of an electrolyte which is thus attained will satisfy both the requirements mentioned above, which are essential to a satisfactory theory. On it we may imagine all the ions divided into pairs formed of ions which are temporarily nearest together, the individuals of each pair undergoing continual change. Between the ions of each pair electrical force exists, which in many ways is similar to a chemical bond, but is different in others. Thus, with chemical association, an ion is either free or combined, it cannot be both together, but here it possesses simultaneously characteristics of both conditions. The ions in each pair, for instance, will be separated spatially from each other nearly as widely as if they were quite free; they are free (e. g. to carry current or exert ionic pressure) in a fraction 
of the cases in which the pair is observed, while they act as combined (exert a molecular pressure) in the remainder. It seems to me that it is a theory on lines similar to these which will ultimately succeed in reconciling all the difficulties connected with strong electrolytes.

\section{SUMmary.}

(1) A critical discussion of the way in which the law of mass action fails for strong electrolytes leads to the conclusion that the reduction in the molecular conductivity with increasing concentration must be neribed mainly to a reduction in the mobilities of the ions, and not to a reduction in their number by association into molecules.

(2) A theoretical investigation of the effect of interionic force shows that identical variations with the concentration will be produced in the conductivity and in the osmotic pressure of the "free" ions (as defined on p. 357).

(3) The application of this result to strong electrolytes shows that the variation in the conductivity and the freezingpoint can be best explained by a modification in the view we take of what constitutes association. According to this ions in strong electrolytes are not associated into molecules; they are neither completely associated nor completely free, but pairs of ions which are temporarily nearest together, in consequence of the electric forces between them, will, in a fraction of cases, act as if bound together, and in the remaining cases as if free.

The University, Sheffield,

December 1917.

XL. Bessel Functions of Equal Order and Argument. By G. N. Watson, M.A., D.Sc., Assistant Professor of Pure Mathematics at University College, London *.

1. A PPRUXIMATE formulæ for the Bessel function $A$ and its derivate, $\mathrm{J}_{n}(n)$ and $\mathrm{J}_{n}{ }^{\prime}(n)$, (when $n$ is large) have been discussed in numerous papers during the last fow years $\dagger$; several of these papers have appeared in this Magazine.

* Communieated by the Author.

† Debye, Math. Ann. lxvii. pp, 535-558 (1909). Rayleigh, Phil. Mag. Dec. 1910. Nicholson, Phil. Mag. Dec. 1907, Aug. 1908, Feb. 1910. Watson, Proc. London Math. Soc. (2) xvi. pp. 150-174 (1917); Proc. Camb. Phil. Soc. xix. pp. 42-48 (1917). Various numerical results have also been given by Airey in a series of recent papers in the Phil. Mag. 